\title{
Aplastic or twig-like middle cerebral artery harboring unruptured cerebral aneurysms treated by clipping and bypass surgery: illustrative case
}

\author{
Ayako Takarada, MD, ${ }^{1}$ Kiyoyuki Yanaka, MD, PhD, ${ }^{1}$ Kuniyuki Onuma, MD, ${ }^{1}$ Kazuhiro Nakamura, MD, PhD, ${ }^{1}$ \\ Nobuyuki Takahashi, MD, $\mathrm{PhD},{ }^{2}$ and Eiichi Ishikawa, $\mathrm{MD}, \mathrm{PhD}^{3}$
}

Departments of ${ }^{1}$ Neurosurgery and ${ }^{2}$ Radiology, Tsukuba Memorial Hospital, Tsukuba, Ibaraki, Japan; and ${ }^{3}$ Department of Neurosurgery, Graduate School of Comprehensive Human Sciences, University of Tsukuba, Tsukuba, Ibaraki, Japan

\begin{abstract}
BACKGROUND Aplastic or twig-like middle cerebral artery (Ap/T-MCA) is a congenital MCA anomaly. It may present with symptoms of both hemorrhage and ischemia, similar to moyamoya disease, and hemodynamic stress may play an essential role in the development of symptoms in both clinical entities. The optimal treatment remains controversial in symptomatic patients with Ap/T-MCA. This report discussed the treatment method for a patient with Ap/TMCA with unruptured aneurysms who presented with intraventricular hemorrhage (IVH) treated by aneurysm clipping and bypass surgery.

OBSERVATIONS In a 46-year-old woman with a sudden headache, computed tomography showed left IVH. Magnetic resonance angiography showed a left MCA aneurysm and MCA trunk stenosis. Three-dimensional angiography demonstrated a plexiform arterial network and multiple aneurysms arising from the MCA and in the plexiform network, leading to the diagnosis of Ap/T-MCA harboring unruptured aneurysms. The patient was successfully treated by craniotomy with aneurysm clipping and bypass surgery to prevent further intracranial hemorrhages and/or aneurysm rupture.
\end{abstract}

LESSONS Especially in cases such as Ap/T-MCA, in which hemodynamic stress has a significant effect, the optimal treatment method should be based on vascular morphology and the impact of hemodynamic stress.

https://thejns.org/doi/abs/10.3171/CASE21360

KEYWORDS aneurysm; hemodynamic stress; middle cerebral artery; twig

Aplastic or twig-like middle cerebral artery (Ap/T-MCA) is a congenital MCA anomaly in which the MCA trunk is either stenotic or occluded with a collateral plexiform network. ${ }^{1-10}$ Ap/T-MCA has been reported in $0.11 \%$ to $1.17 \%$ of all angiographic cases, showing a high prevalence in East Asia. ${ }^{1,3,6,11}$ Approximately $70 \%$ of patients with Ap/T-MCA develop hemorrhagic stroke, 20\% develop ischemic stroke, and only a few are asymptomatic. ${ }^{1-3,6-22}$ These symptoms and hemodynamics resemble moyamoya disease (MMD). Patients with Ap/T-MCA who have hemorrhagic stroke often have aneurysms, as seen in 23 of $31(74.2 \%)$ previously reported hemorrhagic stroke cases. ${ }^{1-3,6-11,13-22}$ In these patients, the optimal treatment remains controversial because it is necessary to consider both the aneurysm location and hemodynamic stress effect due to vascular malformation.
In this article, we report a case of Ap/T-MCA with unruptured aneurysms that presented with an intraventricular hemorrhage (IVH) treated by aneurysm clipping and bypass surgery. In addition, relevant literature is reviewed.

\section{Illustrative Case}

A 46-year-old nonhypertensive woman presented to a local hospital with a sudden severe headache. Computed tomography (CT) showed IVH (Fig. 1). No subarachnoid hemorrhage was observed. The patient was treated conservatively for 2 weeks before referral to our hospital for further examination and treatment. On admission, she had no neurological deficits. IVH had already disappeared on $\mathrm{CT}$ and magnetic resonance imaging, but magnetic resonance angiography showed a left MCA aneurysm. The distal part of the MCA

ABBREVIATIONS Ap/T-MCA = aplastic or twig-like middle cerebral artery; $C T$ = computed tomography; IVH = intraventricular hemorrhage; MMD = moyamoya disease; STA = superficial temporal artery.

INCLUDE WHEN CITING Published August 30, 2021; DOI: 10.3171/CASE21360.

SUBMITTED June 16, 2021. ACCEPTED June 24, 2021.

(c) 2021 The authors, CC BY-NC-ND 4.0 (http://creativecommons.org/licenses/by-nc-nd/4.0/). 


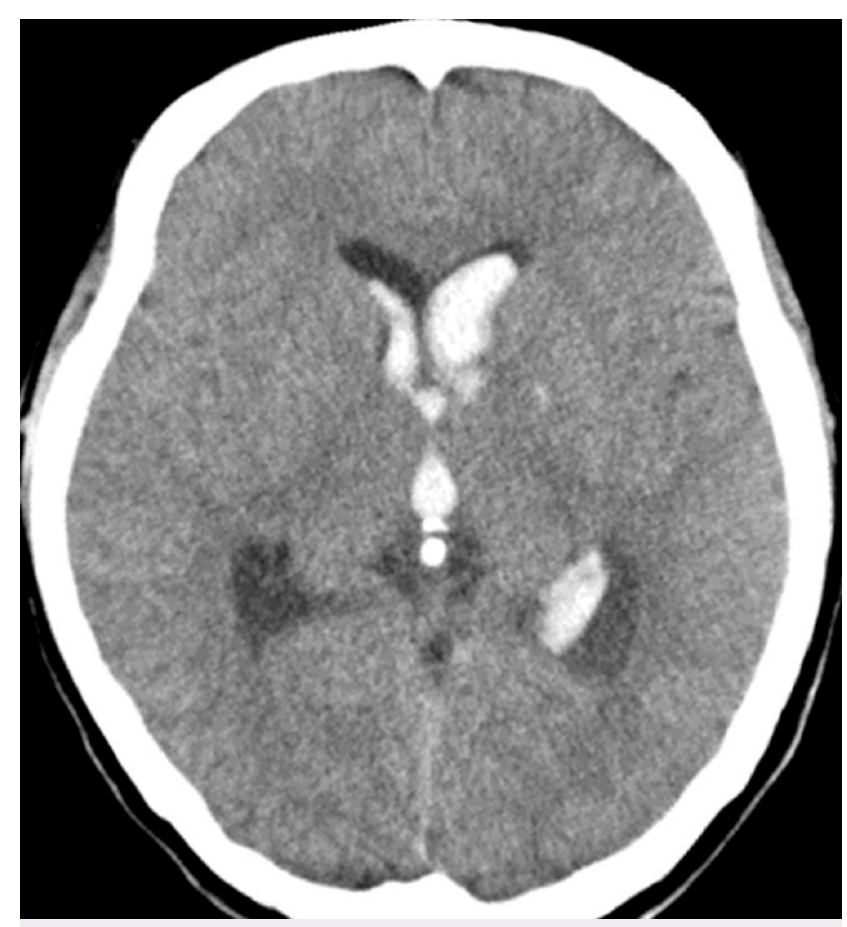

FIG. 1. CT scan showing left IVH.

was visualized incompletely, suggesting severe stenosis just distal to the aneurysm (Fig. 2). Digital subtraction angiography of the left internal carotid artery showed an MCA aneurysm and an abnormal vascular network (Fig. 2). The vessel structure of the distal portion was normal, but delayed blood flow to the periphery was observed. There was no transdural anastomosis. Three-dimensional angiography demonstrated a plexiform arterial network and multiple aneurysms, the largest of which was $5.5 \mathrm{~mm}$ in diameter (Fig. 3), arising from the MCA and within the plexiform network. The diagnosis of Ap/T-MCA harboring unruptured aneurysms was made. In this patient, IVH appeared to be the result of adverse effects of hemodynamic stress caused by the plexiform network. Hemodynamic stress was also considered to be involved in the development of the aneurysms in the plexiform network.
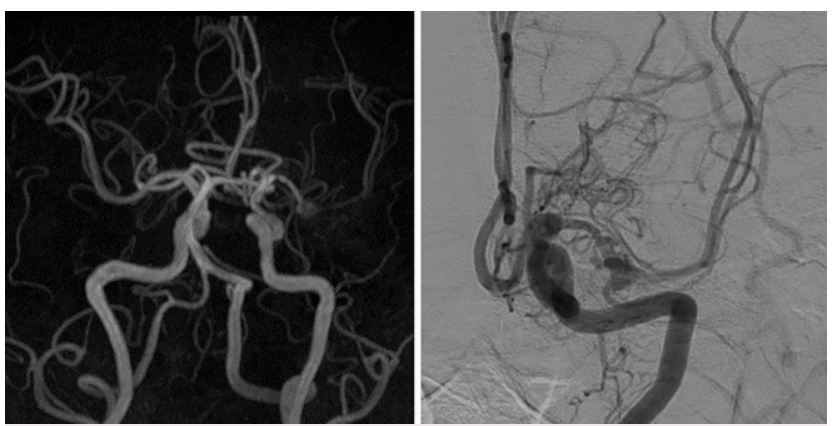

FIG. 2. Magnetic resonance angiography showing an aneurysm arising from the MCA. Severe stenosis of the MCA is also demonstrated (left). Digital subtraction angiogram of the left internal carotid artery showing the aneurysm and the plexiform network. The vessel structure of the distal portion is normal (right).

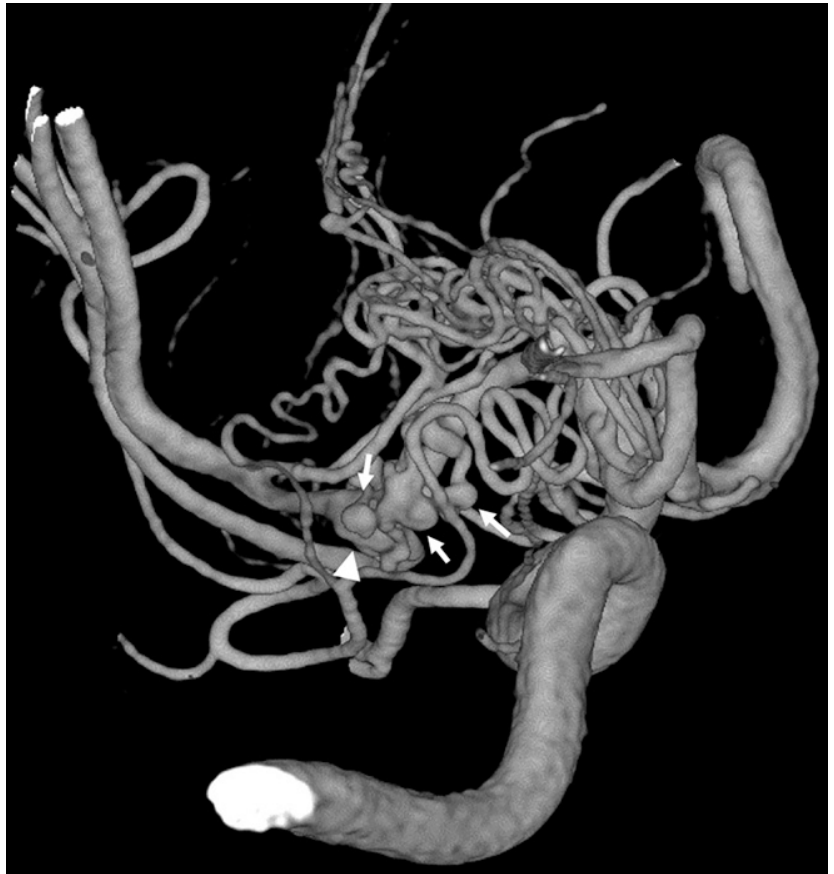

FIG. 3. Three-dimensional angiogram showing the plexiform arterial network and multiple aneurysms arising from the MCA and in the plexiform network (arrow). The largest aneurysm is behind the plexiform network (arrowhead).

The patient received craniotomy with aneurysm clipping and superficial temporal artery (STA) MCA bypass to prevent further intracranial hemorrhaging and future aneurysm rupture. Some of the blood vessels inside the plexiform network showed aneurysmal dilation. All aneurysms were angioplastically obliterated by clipping without reducing blood flow to the distal portion of the MCA. Intraoperative indocyanine green fluorescence angiography showed that all vessels forming the plexiform network remained patent after

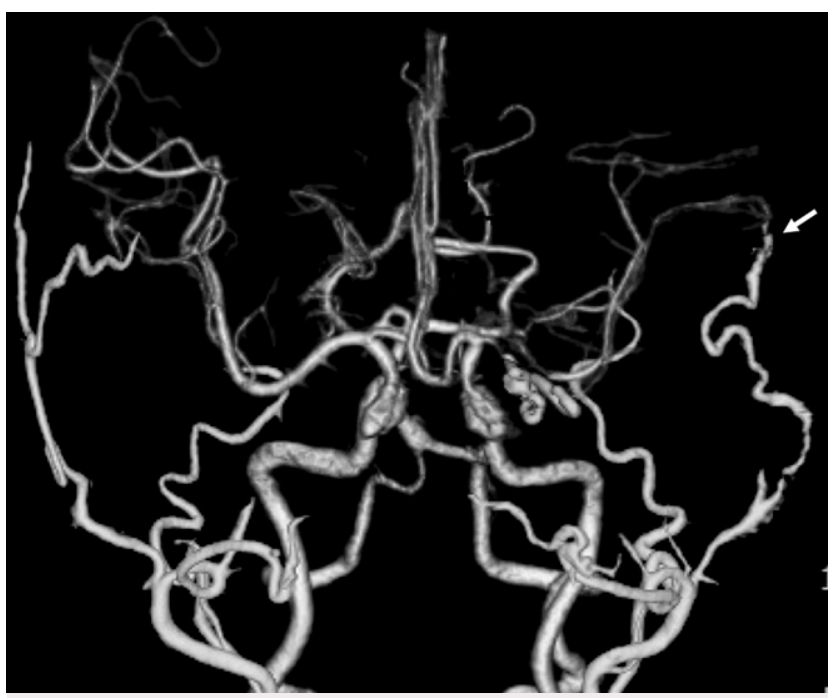

FIG. 4. Postoperative three-dimensional CT angiogram showing the disappearance of all aneurysms and the patency of the bypass (arrow). 
aneurysm clipping, whereas motor evoked potential monitoring showed no waveform alterations throughout the surgery.

The postoperative course was uneventful. Postoperative threedimensional CT angiography verified bypass patency and the disappearance of all aneurysms (Fig. 4). The patient was discharged without any neurological deficits, and her condition has remained unchanged over several years of follow-up. At 6 months after surgery, angiography showed that the aneurysms had disappeared, but the plexiform network remained unchanged (Supplemental Fig. 1).

\section{Discussion}

Ap/T-MCA is a rare anomaly in which the main trunk of the MCA is known to be underdeveloped and has a twig-like, persistent primitive plexus of the MCA. ${ }^{6}$ To the best of our knowledge, approximately 60 cases of Ap/T-MCA have been reported so far. ${ }^{1-3,6-22}$ Of these, 26 cases were associated with aneurysms, including ours (Table 1), with 19 ruptured and 7 unruptured cases. The average age (26 cases) was 54.2 years, and women were more likely to be affected. Half of the ruptured aneurysms developed inside the plexiform network; in contrast, most unruptured aneurysms developed outside or on the opposite side of the plexiform network. In patients with Ap/T-MCA who have ruptured cerebral aneurysms, clipping was mainly performed, but there were some combination bypass and clipping cases as well. Patients had varying prognoses that seemed to depend on the degree of brain damage that occurred when the aneurysm ruptured.

When treating patients with Ap/T-MCA who have aneurysms, the optimal treatment method depends on whether the aneurysm is ruptured and the positional relationship between the aneurysm and the plexiform network. When an aneurysm develops outside the plexiform network, it should be managed using standard treatment indications and methods, regardless of rupture status.

In ruptured aneurysm cases, treatment requires careful consideration, especially if the aneurysm is inside the plexiform network. There are several reports of parent artery occlusion by intravenous embolization of the ruptured aneurysms in patients with Ap/TMCA. ${ }^{2,14}$ Aneurysms that developed inside the plexiform network may differ from common saccular aneurysms; the vessels that form plexiform networks themselves may show aneurysmal dilation, as in our case. It may be unavoidable to sacrifice the parent vessel in

TABLE 1. Summary of cases with aplastic or twig-like middle cerebral arteries associated with aneurysms

\begin{tabular}{|c|c|c|c|c|c|c|}
\hline \multicolumn{2}{|c|}{ Case No./Authors \& Year } & Age/Sex & Clinical Presentation & Aneurysm Location & Treatment & GOS \\
\hline \multicolumn{7}{|c|}{ Ruptured aneurysms } \\
\hline 1 & Fukawa et al., $1981^{13}$ & $16 / F$ & $\mathrm{IVH}$ & Outside the twig & Conservative & PVS \\
\hline 2 & Park et al., $2004^{11}$ & $74 / F$ & $\mathrm{ICH}, \mathrm{SAH}$ & Inside the twig & Clipping & $\mathrm{N} / \mathrm{A}$ \\
\hline 3 & Liu et al., $2005^{3}$ & $67 / F$ & $\mathrm{SAH}, \mathrm{ICH}$ & Inside the twig & Clipping & $\mathrm{N} / \mathrm{A}$ \\
\hline 4 & & $44 / \mathrm{M}$ & SAH, IVH, ICH & Inside the twig & Clipping & $N / A$ \\
\hline 5 & Cekirge et al., $2005^{2}$ & 32/M & SAH, IVH & Outside the twig & Coiling & GR \\
\hline 6 & Kim et al., $2005^{15}$ & $64 / F$ & SAH & Outside the twig & Clipping & $N / A$ \\
\hline 7 & Rodriguez-Hernández et al., $2011^{10}$ & $52 / \mathrm{M}$ & $\mathrm{ICH}$ & Inside the twig & Trapping \& bypass & $M D$ \\
\hline 8 & Seo et al., $2012^{6}$ & 73/M & SAH & Inside the twig & Clipping & $\mathrm{N} / \mathrm{A}$ \\
\hline 9 & Shin et al., $2014^{7}$ & $46 / F$ & SAH, ICH, IVH & Inside \& outside the twig & Clipping & SD \\
\hline 10 & & $49 / F$ & SAH, ICH, IVH & Inside the twig & Proximal occlusion & GR \\
\hline 11 & & 42/M & SAH, ICH, IVH & Inside the twig & Clipping \& trapping & PVS \\
\hline 12 & & 26/M & IVH & Outside the twig & Conservative & GR \\
\hline 13 & Uchiyama, $2017^{9}$ & $77 / F$ & SAH & Inside the twig & $\mathrm{N} / \mathrm{A}$ & $N / A$ \\
\hline 14 & Lang et al., $2017^{16}$ & $61 / \mathrm{M}$ & $\mathrm{ICH}, \mathrm{IVH}$ & Inside the twig & Trapping & GR \\
\hline 15 & & $53 / F$ & SAH & Inside the twig & Clipping & SD \\
\hline 16 & Seno et al., $2017^{22}$ & $49 / F$ & SAH, IVH & Inside the twig & Clipping \& bypass & GR \\
\hline 17 & Sakai et al., $2005^{20}$ & $65 / F$ & $\mathrm{ICH}, \mathrm{IVH}$ & Inside the twig & Clipping \& bypass & SD \\
\hline 18 & Fukuda et al., $2018^{14}$ & $60 / F$ & SAH & Outside the twig & Coiling & $M D$ \\
\hline 19 & Fukuyama et al., $2020^{21}$ & $53 / F$ & SAH & Inside \& outside & Clipping & SD \\
\hline \multicolumn{7}{|c|}{ Unruptured aneurysms } \\
\hline 20 & Seo et al., $2012^{6}$ & $58 / \mathrm{M}$ & Incidental & Outside the twig & Coiling & $N / A$ \\
\hline 21 & & $58 / F$ & $\mathrm{Cl}$ & Outside the twig & Conservative & $N / A$ \\
\hline 22 & & 73/M & SAH (nonaneurysmal) & Outside the twig & Conservative & $N / A$ \\
\hline 23 & & $49 / F$ & $\mathrm{ICH}$ & Outside the twig & EDAS \& coiling & $\mathrm{N} / \mathrm{A}$ \\
\hline 24 & Akkan et al., $2015^{1}$ & $54 / \mathrm{M}$ & Incidental & Outside the twig & Conservative & $\mathrm{N} / \mathrm{A}$ \\
\hline 25 & Yamada et al., $2020^{18}$ & 68/M & $\mathrm{Cl}, \mathrm{ICH}$ & Outside the twig & Bypass & GR \\
\hline 26 & Present case, 2021 & $46 / F$ & IVH & Inside the twig & Clipping \& bypass & GR \\
\hline
\end{tabular}

$\mathrm{Cl}=$ cerebral infarction; EDAS = encephalo-duo-arterio-synangiosis; $\mathrm{GOS}=$ Glasgow Outcome Scale; GR = good recovery; ICH = intracerebral hemorrhage; $\mathrm{MD}=$ moderate disability; N/A = not applicable; PVS = persistent vegetative state; $S A H=$ subarachnoid hemorrhage; $S D=$ severe disability. 
some cases, but maintaining blood flow to the distal portion is especially important in conditions such as Ap/T-MCA, in which hemodynamic stress is involved. Therefore, angioplastic clipping of the aneurysm is considered the most reasonable method. When parent artery occlusion or aneurysm trapping is unavoidable, additional bypass should be considered.

In unruptured cases, treatment to prevent future ruptures should focus on the fragility of the parent vessels, especially if the aneurysm develops inside the plexiform network, because from an embryological point of view, the vessels forming the plexiform network and the aneurysm are considered vulnerable..$^{2,3,6,11}$ It has also been reported that a conservatively managed unruptured aneurysm associated with Ap/T-MCA ruptured several years later. ${ }^{23}$ Although the aneurysms were unruptured in our case, one was relatively large and some others were located within the plexiform network. The risk of future rupture was considered high, and angioplastic clipping to occlude the aneurysms while maintaining blood flow to the distal portion of the MCA was performed. Therefore, if there is a risk of excessive hemodynamic stress, clipping is a valid treatment option even if the aneurysm is unruptured.

An STA-MCA bypass was also performed in our case. Ap/T-MCA and MMD have similar pathologies, and hemodynamic stress plays a major role in both clinical entities. Long-term hemodynamic stress to the collateral vessels is thought to induce vascular abnormalities, leading to hemorrhage in MMD, and direct bypass surgery proved effective in preventing hemorrhagic events by reducing such stress. ${ }^{6,24,25}$ When considering the optimal treatment for patients with Ap/T-MCA, it is necessary to understand the type of stroke and effects of hemodynamic stress, as in MMD cases. IVH in our patient was thought to be caused by such excessive pressure, resulting in the disruption of collateral periventricular vessels. Bypass surgery in patients with Ap/T-MCA to decrease pressure on the fragile vessels and prevent future hemorrhagic stroke has been reported. ${ }^{5,19,22}$ Therefore, reducing hemodynamic stress on aneurysms and fragile plexiform vessels may help prevent future hemorrhaging and ischemia as well as the development of de novo aneurysms. So far, there have been three reports of changes in the plexiform network after bypass surgery, ${ }^{18,20,22}$ with no changes in one case ${ }^{20}$ and regression of the plexiform network in two cases. $^{18,22}$ Postoperative angiography was performed relatively early in our case, so it might be difficult to determine the effect of the bypass on Ap/T-MCA accurately. Follow-up angiography will help to determine the effectiveness of treatment and understand the natural history of Ap/T-MCA. The optimal treatment should take into account not only vascular morphology but also the effects of hemodynamic stress. Further detailed clinical studies with large series of patients with follow-up angiography are required to determine the optimal treatment for patients with Ap/T-MCA with aneurysms.

\section{Observations}

We reported a case of Ap/T-MCA associated with unruptured aneurysms that was successfully treated by clipping and bypass surgery. Especially in cases featuring Ap/T-MCA, in which hemodynamic stress has a significant effect, optimal treatment depends on vascular morphology and the effect of hemodynamic stress.

\section{Lessons}

Especially in cases such as Ap/T-MCA, in which hemodynamic stress has a significant effect, the optimal treatment method should be based on vascular morphology and the impact of hemodynamic stress.

\section{Acknowledgments}

We would like to thank Dr. Alexander Zaboronok of the University of Tsukuba Faculty of Medicine, Department of Neurosurgery, for professional and language revision and Dr. Bryan J. Mathis of the University of Tsukuba Hospital International Medical Center for language revision.

\section{References}

1. Akkan K, Ucar M, Kilic K, Celtikci E, Ilgit E, Onal B. Unfused or twig-like middle cerebral artery. Eur J Radiol. 2015;84(10): 2013-2018.

2. Cekirge HS, Peynircioglu B, Saatci I. Endovascular treatment of an "anterior cerebral artery" aneurysm in a patient with "embryonic unfused middle cerebral artery" anomaly: a case report. Neuroradiology. 2005;47(9):690-694.

3. Liu HM, Lai DM, Tu YK, Wang YH. Aneurysms in twig-like middle cerebral artery. Cerebrovasc Dis. 2005;20(1):1-5.

4. Lutz T, Mönnings P, Ayzenberg I, Lukas C. Twig-like middle cerebral artery: a seldom vessel anomaly of important relevance. Clin Neuroradiol. 2018;28(3):441-443

5. Matsunaga Y, Izumo T, Morofuji Y, Horie N, Hayashi K, Matsuo T. Revascularization for aplastic or twiglike middle cerebral artery: a case report. J Stroke Cerebrovasc Dis. 2018;27(5):e78-e79.

6. Seo BS, Lee YS, Lee HG, Lee JH, Ryu KY, Kang DG. Clinical and radiological features of patients with aplastic or twiglike middle cerebral arteries. Neurosurgery. 2012;70(6):1472-1480.

7. Shin HS, Lee SH, Ryu CW, Koh JS. Flow-related intracranial aneurysms associated with unfused arterial twigs relevant to different vascular anomalies: embryologic and hemodynamic considerations. Acta Neurochir (Wien). 2014;156(9):1637-1646.

8. Tashiro R, Inoue T, Shibahara I, et al. Nonaneurysmal subarachnoid hemorrhage due to unfused or twiglike middle cerebral artery rupture: two case reports. J Stroke Cerebrovasc Dis. 2016;25(6): e77-e78.

9. Uchiyama N. Anomalies of the middle cerebral artery. Neurol Med Chir (Tokyo). 2017;57(6):261-266.

10. Rodríguez-Hernández A, Lu DC, Miric S, Lawton MT. Aneurysms associated with non-moyamoya collateral arterial networks: report of three cases and review of literature. Neurosurg Rev. 2011;34(4): 517-522.

11. Park J, Hwang JH, Hamm IS. Aneurysm rupture at an anomalous collateral artery that extended from the proximal A2 segment to the middle of the M1 segment, bypassing atresia of the internal carotid artery bifurcation. Case report. J Neurosurg. 2004;100:332-334.

12. Edgell RC, Boulos AS, Borhani Haghighi A, Bernardini GL, Yavagal DR. Middle cerebral artery stenosis associated with moyamoya pattern collateralization. Front Neurol. 2010;1:119.

13. Fukawa $\mathrm{O}$, Aihara $\mathrm{H}$, Ishii $\mathrm{K}$, et al. Middle cerebral artery occlusion with moyamoya phenomenon. In: First Report: Clinical Course and Angiographical Findings. 10th Conference of Surgery for Cerebral Stroke, Tokyo, Japan. 1981;36-41.

14. Fukuda $Y$, Matsunaga $Y$, Hirayama K, et al. A case of aplastic or twig-like middle cerebral artery associated with a ruptured $A 1$ aneurysm at the origin of the anomalous collateral artery. Nosotchu. 2018;40(2):75-80.

15. Kim MS, Oh CW, Hur JW, Lee JW, Lee HK. Aneurysms located at the proximal anterior cerebral artery and anterior communicating artery associated with middle cerebral artery aplasia: case report Surg Neurol. 2005;64(6):534-537.

16. Lang M, Moore NZ, Witek AM, Kshettry VR, Bain MD. Microsurgical repair of ruptured aneurysms associated with moyamoya-pattern collateral vessels of the middle cerebral artery: a report of two cases. World Neurosurg. 2017;105:1042.e5-1042.e10. 
17. Rivera R, Sordo J, Badilla L, Bravo E, Riveros R, Giacaman P. Middle cerebral artery occlusion with moyamoya-like vessels and aneurysms. A report of two cases. Interv Neuroradiol. 2014;20(1): 96-99.

18. Yamada D, Ishibashi R, Kinosada M, et al. Aplastic or twig-like middle cerebral artery with short-term ischemia and bleeding. Japanese Journal of Stroke. 2020;42(3):190-195.

19. Inoue R, Katayama S, Kasai N, Hori S. Middle cerebral artery occlusion with unilateral moyamoya like vessels and with ruptured anterior cerebral artery aneurysm: its relation to the antiphospholipid antibody syndrome. Article in Japanese. No To Shinkei. 1994;46(10):995-998.

20. Sakai K, Mizumatsu S, Terasaka K, Sugatani H, Higashi T. Surgical treatment of a lenticulostriate artery aneurysm. Case report. Neurol Med Chir (Tokyo). 2005;45(11):574-577.

21. Fukuyama R, Yamamura K, Murata $\mathrm{H}$, Miyatake $\mathrm{S}$, Matsumoto $\mathrm{N}$, Abe $\mathrm{H}$. Ruptured aneurysm of an aplastic or twig-like middle cerebral artery with ring finger protein 213 mutation: a case report. Article in Japanese. No Shinkei Geka. 2020;48(6):533-540.

22. Seno T, Kohno K, Tanaka H, et al. A case of ruptured distal anterior choroidal artery aneurysm associated with a twig-like middle cerebral artery, treated with single-stage aneurysm clipping and STA-MCA double anastomoses in the acute phase. Article in Japanese. No Shinkei Geka. 2017;45(8):691-697.

23. Uchiyama T, Okamoto H, Koguchi M, Tajima Y, Suzuyama K. A case of aplastic or twig-like middle cerebral artery presenting with an intracranial hemorrhage two years after a transient ischemic attack. Article in Japanese. No Shinkei Geka. 2016;44(2):143-148.

24. Inoue A, Kohno K, Fukumoto $S$, et al. A case of ECA-MCA double anastomoses for hemorrhagic type of twig-like MCA. Article in Japanese. No Shinkei Geka. 2016;44(6):463-471.
25. Miyamoto S, Yoshimoto T, Hashimoto N, et al. Effects of extracranial-intracranial bypass for patients with hemorrhagic moyamoya disease: results of the Japan Adult Moyamoya Trial. Stroke. 2014:45(5):1415-1421.

\section{Disclosures}

The authors report no conflict of interest concerning the materials or methods used in this study or the findings specified in this paper.

\section{Author Contributions}

Conception and design: Takarada, Yanaka, Nakamura. Acquisition of data: Takarada, Yanaka, Nakamura, Takahashi. Analysis and interpretation of data: Takarada, Yanaka, Takahashi. Drafting the article: Takarada, Yanaka. Critically revising the article: Takarada, Yanaka Reviewed submitted version of manuscript: Ishikawa, Takarada, Onuma, Nakamura. Approved the final version of the manuscript on behalf of all authors: Ishikawa. Statistical analysis: Takarada.

Administrative/technical/material support: Takarada. Study supervision: Ishikawa, Takarada, Nakamura.

\section{Supplemental Information}

Online-Only Content

Supplemental material is available with the online version of the article. Supplemental Fig. 1. http://thejns.org/doi/suppl/10.3171/CASE21360.

\section{Correspondence}

Eiichi Ishikawa: University of Tsukuba, Ibaraki, Japan. e-ishikawa@md. tsukuba.ac.jp. 